\title{
« Magicriture » plurielle : un éthos de la survie
}

\section{Malika Hadj Naceur}

\section{CpenEdition}

\section{Journals}

Édition électronique

URL : http://journals.openedition.org/recherchestravaux/408

ISSN : 1969-6434

Éditeur

UGA Éditions/Université Grenoble Alpes

\section{Édition imprimée}

Date de publication : 30 juillet 2010

Pagination : 77-88

ISBN : $978-2-84310-174-8$

ISSN : 0151-1874

Référence électronique

Malika Hadj Naceur, « " Magicriture » plurielle : un éthos de la survie», Recherches \& Travaux [En ligne], 76 | 2010, mis en ligne le 30 janvier 2012, consulté le 07 septembre 2020. URL : http:// journals.openedition.org/recherchestravaux/408 
Malika Hadj NACEUR

Université d'Alger

\section{«Magicriture» plurielle : un éthos de la survie}

«Le livre ivre et libre ira plus loin que la fiction et le dire narratif»

Frankétienne

«Les amateurs d'écriture sont dispersés, clandestins, écrasés par mille contraintes, intérieures même. C'est là un problème de civilisation : mais, pour moi, ma conviction profonde et constante est qu'il ne sera jamais possible de libérer la lecture si, d'un même mouvement, nous ne libérons pas l'écriture.»

Roland Barthes

Littérature réfractaire, littérature créative, littérature inventive bruissante de questionnements et d'une émotion stimulatrice-dissimulatrice dans la réciprocité de la conscience ironisante et de la conscience ironisée qui l'infiltrent : ainsi se dessinent, schématiquement, les contours de la littérature maghrébine de ces dernières décennies. Les «magicritures ${ }^{\mathrm{I}}$ » maghrébines invitent à l'exploration des textes et, au delà, à la rencontre du monde propre qui

I. Le néologisme est de Frankétienne. Je le lui emprunte, consciente cependant que les écritures auxquelles je me réfère n’ont pas, compte tenu du contexte culturel différent, la même dimension spécifiquement résurrectionnelle de l'art. Elles en conservent néanmoins des échos familiers dans l'option faite à la stratégie fictionnelle du mentir-vrai (grossissement requis des faits réels qui nourrissent l'imaginaire, chemins narratifs entrecroisés, transfiguration du tragique...) et l'ambition de nommer un monde qui tend à un degré supérieur d'existence. 
les "parle», des ressources culturelles qui les travaillent, qui plaident pour les siens et pour soi, sa manière propre, pour ainsi dire, de remettre la réalité sur ses pieds, de donner sens et fertilité à la malédiction apparente du vécu. Dans nombre d'œuvres, en effet, la recherche esthétique l'emporte sur le dévoilement du réel, des turbulences du quotidien. L'insécurité, le chaos, le cri deviennent un principe dynamique de créativité, jeu(x) et enjeu(x) d'une observation lucide et revisitée du présent pour y creuser les voies buissonnières d'un futur à hauteur d'homme armé contre le désespoir, d'un espace neuf de l'écriture. Et ce rêve dans l'ancrage de l'actualité tragique se donne à lire, stratégiquement, dans de nombreuses «formes-sens» parmi lesquelles, dans le cadre de cet article, j'opérerai un choix nécessairement limité.

\section{Leitmotive obsédants}

Comme les auteurs de l'Afrique subsaharienne dont les écrits continuent de remettre en cause des États "nouveaux", les écrivains maghrébins évoquent, à partir du lieu où ils vivent, avec insistance, la question de la responsabilité des dirigeants, égratignant au passage, de plus en plus, le peuple qui se nourrit passivement d'une culture de l'impunité et du désespoir dans une société «qui marche sur la tête ${ }^{2}$.

Parmi les meurtrissures marquantes du vécu socio-politique, celles de l'imposture étatique pseudo-démocratique et de l'obsession d'un ailleurs réparateur qui lui est corollaire n'en finissent pas d'inspirer les auteurs. La littérature, surtout algérienne et marocaine, se fait largement l'écho des invariants de l'écriture de l'Histoire de ces pays, dans un tout autre contexte cependant que celui des débuts de l'Indépendance. De nombreux écrits convoquent ces questions, mettent en images, sur fond de violence multiforme et tragiquement familière, le chaos, l'échec des politiques de développement, l'envers du discours propagandiste des «chefs». Dans la lignée des prédécesseurs de renom ${ }^{3}$ qui ont marqué leur époque et dont les écrits attestaient déjà d'un imaginaire en liberté, d'autres auteurs, partant du constat que décidément l'humanité tourne mal, entreprennent de conjurer les figures menaçantes du présent au moyen d'une esthétique de la libération (associant l'art, le jeu et la transgression).

Ainsi, dans plusieurs œuvres d'Assia Djebar', écrivaine à la production abondante, l'écriture se laisse guider par la spirale des voix et des regards qui

2. Selon la formule significative du narrateur de Tombéza de R. Mimouni.

3. Pour la plupart aujourd'hui disparus : le M. Dib de la seconde période, M. Mammeri, Y. Kateb, R. Mimouni, D. Chraibi... pour ne citer que quelques auteurs phares.

4. Entrée à l'Académie française le 16 juin 2005. 
s'entrecroisent et se confondent avec le dire propre de la narratrice. Celle-ci écrit dans Vaste est la prison ${ }^{5}$ :

Apprendre à voir, je l'ai découvert, c'est se ressouvenir certes, c'est fermer les yeux pour réécouter les chuchotements d'avant $[\ldots]$ c'est rechercher les ombres qu'on croit mortes. ( $V$, p. 298)

Car «le regard sur le dehors est en même temps retour à la mémoire», «regard réflexif sur le passé [qui] pouvait susciter une dynamique pour une quête sur le présent, un avenir à la porte». Cette écriture qui enchaîne les regards (celui d'Assia Djebar, l'historienne de formation, celui de l'Algérienne narratrice épouse-mère-écrivaine, celui de la cinéaste qu’elle est aussi), relate des «allées et venues de femmes fuyantes du passé lointain ou récent» $(V$, p. 172) appartenant à ce "peuple des cloîtrées d'hier et d'aujourd'hui» ( $V$, p. 174). Cette écriture lie le «voir» au «dire» et à l'« écrire»:

Qu'est-ce que "tourner» pour moi, sinon tenter de regarder à chaque fois du premier regard, d'écouter de la première écoute? ( $V$, p. 200)

D’où l'emboîtage des séquences, ces "circonvolutions en désordre" qui tissent l'imaginaire et le fécondent. Il s'agit donc d'«avertir de la fracture du monde", de nommer la souffrance tue de ces "fugitives " parmi lesquelles elle se compte, celle aussi de cette "Algérie chasseresse [...] avalée» ( $V$, p. 348). Cette "chasse aux images $(V \text {, p. } 174)^{6} »$ renouvelle la facture du récit en dessinant des cheminements de sens croisés, enlacés, marqueurs d'une parole fortement connotée en communion avec le monde de «toutes les femmes de tous les harems» $(V$, p. 174), de tous les disparus :

Oh, je vois (ou j'entends, je ne sais) je vois les morts les plus anciens [...], je les vois, les morts de très loin, non parce que je prétends à l'héritage de la magicienne Lala Rhia (je n'ai ni brasero autour de mes jupes, et je n'irai en nul pèlerinage, non), simplement parce que, ces deux ou trois dernières saisons, en mon pays, tous les morts, indistinctement, reviennent. ( $V$, p. 335)

Et ce télescopage quasi constant des «cris» d'où qu'ils proviennent (mobilité labyrinthique des espaces revisités) et quel que soit l'écho qu'ils répercutent ( "la débâcle de la fête guerrière d'hier, de l'horreur indicible d'aujourd'hui», $V$, p. 339) recadre la tyrannie mémorielle pour «faire entendre. Et de là : se faire entendre.» $(V, \mathrm{p} .37)$.

5. A. Djebar, Vaste est la prison, Albin Michel, Le Livre de poche, (désormais V), 2002.

6. Il s'agit de l'image-réalité, par exemple, des femmes voilées, de ces quelque cinq cents millions de "ségréguées du monde islamique» (p. I74), dont A. Djebar se fait transfuge et auxquelles renvoient les métaphores corporelles : «corps femelle voile», "linge-linceul», «regard miniature», «fente étrange que les puristes photographient parce qu'ils trouvent pittoresque ce petit triangle noir à la place d'un œil »... 
En mettant à contribution différents modes de savoirs (interdits et tabous de la société arabo-musulmane, autobiographie, culture...), en optant pour une technique d'affabulation qui procède de l'emboîtage, l'écrivaine rend plus performant son propos, cette écriture de résistance sans cesse déconstruite par la mise en jeu de dispositifs narratifs à valeur de questionnements. À la narratrice-transfuge de Vaste est la prison, dont les voix et les regards façonnent le texte et plaident ostensiblement pour une mise en scène de la parole identitaire et du verbe créatif qui la met en forme, elle fera dire :

Ainsi, la fiction, à l'intérieur du documentaire conserve un symbole d'espérance. ( $V$, p. 302)

Cette stratégie séductrice, car configuratrice du sens perdu du monde, nous la retrouvons, sous d'autres modulations romanesques ${ }^{7}$, dans Harraga de Boualem Sansal, Partir de Tahar Ben Jelloun, Don Quichotte à Alger de Waciny Laredj, pour ne citer ici que quelques ouvrages qui revisitent l'errance et s'inscrivent dans la perspective d'une écriture au fort pouvoir de suggestion. Cette errance inscrite dans les intitulés mêmes des romans est un autre leitmotiv obsédant de la littérature maghrébine qui se prête aux jeux capricieux de l'imaginaire pour une descente dans le royaume des ombres (les maux du vécu), frayant un passage de l'autre coté du miroir tendu par le réel. Il ressort, en effet, de la lecture de ces textes la volonté des auteurs d'inscrire l'imaginaire dans un espace fictionnel où se meuvent des personnages qui, selon une trajectoire oscillant entre fuite et quête, voyage et conquête, narrent un univers chaotique dominé par une seule issue : la fuite. Fuite déguisée en voyage-pérégrination reconvoquant le mythe chez Waciny Laredj (son Don Quichotte est "aventurier et chercheur de sens» $(D Q$, p. I70) pour « faire éclater [des] vérités cachées») (DQ, p. I66); fuite réelle des «enfants de la perdition» $(H$, p. 24) chez Tahar Ben Jelloun et Boualem Sansal.

Ces dernières œuvres, parues à une année d'intervalle, traitent très explicitement de l'errance : celle des traversées clandestines massives qui font la une des journaux et celle des circonvolutions du dire fictionnel qui la mettent en forme et lui impriment ses visées esthétiques. L'une et l'autre inscrivent la narration dans des univers dûment nommés, référant très précisément à l'Algérie et au Maroc d'aujourd'hui, présentés sans détours comme lieux de turpitudes quotidiennes : "l'Algérie, un certain tiers-monde qui se mord la queue» (H, p. I46), "Alger est conçue pour perdre son monde [...]» (H, p. I65), «c'est ça le Maroc [...] dans notre pays bien-aimé, la corruption c'est

7. B. Sansal, Harraga, Gallimard, 2005 (désormais H); T. Ben Jelloun, Partir, 2006 (désormais P); W. Laredj, Don Quichotte à Alger, Littérature Action, nº 3I4, sept.-oct., Marsa Éditions, 1996 (désormais $D Q$ ). 
l'air que l'on respire, oui, nous puons $[\ldots]$ » (P, p. I7-I8), "Soigner les apparences et faire des cochonneries en douce, c'est ça le Maroc qui m’énerve" ( $P$, p. 80), etc. Quelque chose, en effet, bouge dans le regard des Maghrébins sur eux-mêmes. Dans un pamphlet où il propose à ses compatriotes de réapprendre à se parler ${ }^{8}$, Boualem Sansal écrit :

Trêve de lamentations, marre des grincements de dents et des sarcasmes, il nous faut parler et, si possible, ne rien nous cacher. C'est le moins que nous puissions faire pour nos enfants, leur laisser une belle image de nous. Démarrer la vie sur des menteries et des rêves creux, soit, mais qu'ils le sachent $(P R, \mathrm{p} .22)$.

La parole oblique n'est plus de mise car «l'amour, le sexe et le tintouin aussi, ça travaille, ça obnubile, ça démolit, ça écorche» ( $H$, p. I29). Les personnages de Tahar Ben Jelloun, quant à eux, vivent des histoires d'exil : ni héros, ni monstre, Azel se présente comme seulement « un homme pris au piège de ses faiblesses» $(P$, p. I5I) ; fille libérée, Kenza échappée du "filet de la fatalité» ( $P$, p. I67) vit sans complexes son amour avec Nazim le Turc; "Zamel», Abdeslam confesse sous d'autres cieux son homosexualité (car «nous sommes comme les autres pays, sauf qu'on ne parle pas de ces choses-là, chez nous on ne va pas à la télé pour avouer qu'on aime les hommes!» $(P$, p. 136)...

Le parcours des candidats à l'immigration sauvage auxquels renvoient les formulations à tonalité tragico-ludique («Harraga» [H, p. 46], «les Zarabes aux épaules mouillées») ( $P$, p. I53) ne découvre pas seulement des tragédies humaines, mais procède aussi de la découverte d'un nouveau monde, celui d'une écriture qui s'accomplit comme processus de contre-leurres et espace d'un "dire» lucide et critique. "Révélateur de la complexité du malheur» ( $P$, p. 243), l'exil donne, en effet, à lire le mouvement d'une conscience interpellative, questionneuse du sens du monde :

Faut-il aimer la vie pour tant endurer. Faut-il aimer la mort pour tant la chercher. D'où vient le mal ? ( $H$, p. 203)

Quelle vie est la vie souterraine? $(H, \mathrm{p} .47)$

Comme on s'en doute, cet examen donne lieu à un regard sur soi peu amène :

Nous sommes musulmans pauvres, sans papiers, donc dangereux [...] on n'est pas un cadeau. (P, p. 158$)$

L'évolution étant ce qu'elle est et le monde musulman ce que nous voyons, j'ai cherché à comprendre pourquoi les filles étaient martyrisées et les garçons adulés et s'il fallait y voir le doigt de Dieu ou la main du Diable ( $H$, p. I24-I25).

8. B. Sansal, Poste restante : Alger-Lettre de colère et d'espoir à mes compatriotes, Gallimard, 2006, (désormais $P R$ ). 
Ce constat sans complaisance et amer fait de l'errance une voie privilégiée de connaissance. Et cet apprentissage, lieu d'une remontée salvatrice, impose l'errance comme quête et conquête de l'ailleurs et de soi, comme aventure périlleuse : "Les Harraga ont inventé pour nous de nouvelles façons de mourir. [...] Quelle vie est la vie souterraine ?» ( $H$, p. 47)

Le voyage intérieur conduit à une traversée douloureuse que les métaphores des textes transmuent en cheminement vivifiant incitant à aller au-delà des apparences pour dépasser la fatalité déprimante du mal et disposer son esprit à croire en l'avènement d'un mieux-être à venir. Cette vision du monde met hors-jeu la mort inexorablement attachée à la thématique de l'émigration clandestine dans sa réalité d'obsession-malédiction :

Partir, partir ! Partir n'importe comment, à n'importe quel prix, se noyer, flotter sur l'eau, le ventre gonflé, le visage mangé par le sel, les yeux perdus... Partir ! (P, p. I49)

Rien de nouveau, le pays continue de se vider comme une baignoire trouée. Tant qu'il y a de la vie, il y aura des morts et des disparus ( $H$, p. I62)

Cette vision a, chez Tahar Ben Jelloun, plusieurs figures. "Toutia», «l'araignée tantôt dévoreuse de chair humaine, tantôt bienfaitrice...» ( $P$, p. I2), est un personnage irréel né de l'imagination désespérée des obsédés de la clandestinité pour qui elle est, au début de leur parcours, la voix appelant au voyage vers la terre promise. Au terme du parcours, alors que la vie ailleurs n'est plus possible, elle revient sous les traits d'une belle jeune femme "tatouée sur le front et le menton" ( $P$, p. 256) (symbole de l'appel des origines) sous le nom de "Toutia-la-Sublime» sur le bateau baptisé de son nom qui ramène les exilés chez eux. Elle seconde le capitaine - «l'homme d'un autre temps» $(P$, p. 256) - dans cette traversée onirique. Sa voix n'est plus alors ensorcelante, prélude à la mort, mais rythmée de chants arabo-andalous pour le retour au bercail de tous les égarés, vivants et morts, de tous ceux aussi convoqués par la mémoire orale et livresque.

Embarquement donc providentiel, chargé d'espérances, de tous les principaux figurants des histoires d'exil. Parmi eux Azel, le personnage central du roman dont le nom complet est tout un programme et qui a payé de sa vie son expérience de l'exil, de "gigolo à domicile9" $(P$, p. 244), de "renégat à son identité et à son sexe» $(P, \mathrm{p} .89)$ et d'indicateur de flics dans la lutte contre le terrorisme en Europe. Son cercueil sur le bateau atteste d'un retour héroïque réparateur, en être qui a accompli sa métamorphose : il y a là un clin d'œil amusé au personnage de Kafka qui préludait à sa destinée :

9. "Azz El Arab", "La fierté, la gloire des Arabes [...] La crème des Arabes ! Celui qui est précieux et bon [...]", p. 49. Son vécu s'avèrera en contradiction avec cette symbolique nominale. 
Il pensait à Kafka et à la métamorphose.

Il ne l'avait pas lu mais il se souvenait d'un cours magistral de son professeur de philo sur le sujet, je vais me transformer, devenir quelqu'un d'autre [...], je passe d'un personnage à un autre... $(P, \mathrm{p} .237)$

Avec les migrants, sur le même bateau, le lecteur retrouve le légendaire et rusé Moha, personnage errant immémorial convoqué ici comme pour donner à la fiction un dénouement fantaisiste et riche d'enseignements visionnaires. Il est cette "parole non discutable», ce "temps hors du temps" ( $P$, p. 255), ce personnage «qui erre sur les routes depuis si longtemps [qui a] perdu [son] nom et [n'a] plus de visage» $(P, \mathrm{p} .257)$, dont la réapparition en «immigré anonyme» $(P$, p. 266) élargit la question de l'errance à celle infinie et symbolique d'un perpétuel refus de la douleur :

[...] partons, voguons sur les mers jusqu'à l'extinction de la plus petite lumière que porte l'âme d'un être, qu'il soit d'ici ou d'ailleurs, qu'il soit un homme de Bien ou un être égaré possédé par le mal, nous suivrons cette ultime lumière, si mince, si fine soit-elle, peut-être que d'elle jaillira la beauté du monde, celle qui mettra fin à la douleur du monde. $(P$, p. 267)

Et les apparitions surnaturelles de Moha travesti en arabe, de Toutia en hôtesse arabe (par son tatouage et ses chants spécifiques), ancrent le rêve de cet ailleurs plus clément dans un retour aux siens et à soi qui en dit long sur la portée du témoignage mis en fiction : la traversée clandestine des désespérés, comme celle, assumée, apaisée, des migrants de tous lieux et en tous temps sur le "bateau magique» ( $P$, p. 260), est métaphore d'un double itinéraire, celui d'une quête de la survie et du bonheur individuel, mais aussi celui, déréalisé, associant lire et voyager en tant que modes d'un désir de transcendance cheminant sous les mots, sous les noms. Dans cet ordre d'idées, l'imaginaire de Tahar Ben Jelloun, comme celui d'ailleurs de Boualem Sansal, se nourrit de réminiscences livresques, de pérégrinations dans une réserve de savoirs qui n'ont rien de gratuit.

Chez le premier, outre Moha, l'incursion de Don Quichotte dont le combat ne prête plus à rire, mais rappelle les idéaux de justice qui y préludaient, celle de Flaubert s'inventant une vie de "personnage magique» ( $P$, p. 262), "un emploi fictif dans une fiction [...] pour ne pas affronter [...] la vraie réalité, celle qui nous colle à la peau» $\left(P, \mathrm{p} .{ }_{2} 63\right)$. Chez le second, le «brin de causette à ces messieurs du passé» ( $H$, p. I75), les clins d'œils aux "belles lectures" ( $H$, p. 36) et au vécu "crue[l] et bête des temps modernes» $(H$, p. 36) qui donnent lieu à des passages énigmatiques et ludiques participent de la mise en forme extravagante de la création romanesque. Celle-ci conçue symboliquement comme ordonnancement du chaos fait du voyage auquel 
convie la lecture une quête de vérité, une progression spirituelle, un désir profond de changement intérieur plus que de déplacement local.

Le dénouement carnavalesque de Partir qui donne à lire le roman comme une fiction flottante - «et si ce bateau n'était qu'une fiction, un roman flottant sur les eaux, un roman en forme de bouteille jetée à la mer par tant de mères éplorées et fatiguées d'attendre ?» $(P$, p. 26I $)$ - entre en relation avec l'Harraga de Boualem Sansal : les interpellations aux fantômes de la vieille demeure de la narratrice, le récit du rêve apocalyptique des «Hominiens cou[rant] vers d'improbables abris» ( $H$, p. I85), le compte rendu du reportage sur les gens sans pays «march[ant] comme des morts» vers «la Terre promise» $(H, \mathrm{p} .189)$, les intermèdes poétiques nombreux ponctuant sporadiquement la narration, manifestent la volonté commune des auteurs de raconter un voyage intérieur qui serait éveil à soi, à une conscience supérieure de la vie conçue comme navigation périlleuse, à une traversée des consciences et de l'existence où le passeur serait l'écrivain.

L'écriture, telle qu'elle s'accomplit ici, est bien le moyen de vaincre la mort et l'oubli, d'être partie prenante dans l'exploration de l'actualité. Ces fictions du délire enseignent, ludiquement, magiquement, à s'assumer «Harraga dans le cour» (H, p. 205), à être réceptif aux paroles de «Moha le fou, Moha le sage» ( $P$, p. I45) descendu de son arbre pour se faire conscience ironique de tous : «si vous entendez des paroles, c'est qu'elles sortent toutes seules, elles prennent le large, elles se libèrent, elles disent la vérité» ( $P$, p. I48).

\section{Dérision de l'acte d'écrire}

De plus en plus, la fiction est traitée de manière à prouver son pouvoir de fiction. Autrement dit, et compte tenu de son statut propre de création, elle entend se donner à lire comme un espace de tous les possibles participant d'un jeu de compossibilitée ${ }^{\circ}$. Compossibilité de l'inintelligible et de l'intelligible, de l'invraisemblable et du vraisemblable qui crée un espace fictionnel qui se donne à lire de manière allusive. Ainsi, dans le roman de Tahar Ben Jelloun, le «je» narrateur de l'Auberge des pauvres" ${ }^{\text {II }}$ qui sollicite d'emblée la complicité du lecteur, choisit-il d'"errer en pays inconnu, sur une terre de fiction» (AP, p. 20) qui s'édifierait au gré des histoires amassées, car, selon les paroles prophétiques de la "vieille» : "il n'y a pas mieux pour dire la vérité, des histoires invraisemblables, des contes effroyables» (AP, p. 48). Au

Io. La compossibilité est entendue donc ici comme adjonction, simultanéité des significations, des orientations créatives de lecture.

II. T. Ben Jelloun, L’Auberge des pauvres, Seuil, 2000 (désormais $A P$ ). 
rythme d'un "Dire» modulé par l'écoute de la vie des autres - «des blessés, des écorchés, des éclopés, des visages façonnés par le malheur» (AP, p. 48) -, le narrateur-écrivain crée un imaginaire composite où se mêlent les destinées, l'horrible - le secret de «l'Auberge des pauvres, lieu de toutes les turpitudes» ( $A P$, p. 260), et des "crânes polis par des mains d'enfants» conservés dans le coffre de la Vieille - et le merveilleux de l'espace-roman-Auberge.

Dans cette création romanesque originale, c'est cette dernière, par ses enseignements, qui l'aide à voir clair en lui ( je pense qu’on se dévoile en racontant les autres»), ( $A P, \mathrm{p} .52)$, à assumer, à l'instar des personnages mis en fiction, son «destin mal apprivoisé », à «sortir de son ancienne peau » $(A P$, p. 268) pour se découvrir autre :

$[\ldots]$ vous m'avez montré le chemin pour me connaître moi-même. (AP, p. 268)

Je rêve d'être à l'origine d'un ouvrage que tout le monde rechercherait et qui n'existerait que dans mon imagination, je serais ce livre [dont] les phrases coulent l'une après l'autre et viennent se ranger dans d'autres yeux, dans d'autres regards, comme si j'étais la mémoire initiale, celle qui raconte à l'infini l'histoire de cette illusion magique. ( $A P$, p. 262-263)

Par la grâce d'une fiction s'autoélaborant, par le «pouvoir des mots et des images" ( $A P, \mathrm{p} .34)$, se joue une mise en scène ludique et critique du monde qui place l'homme en face d'un miroir (le roman et ses histoires) où il découvre l'être qu'il dissimule.

\section{Compossibilité des rôles}

Abdelatif Laâbi, dans Les Rides du lion ${ }^{12}$, a exploré plus encore l'expérience $\mathrm{du}$ "je» narrateur faisant participer le lecteur à l'élaboration du roman. Le dialogue avec sa muse est, en effet, le fil rouge d'un parcours énonciatif signifiant que le roman se prend pour sujet de l'écriture. En donnant la parole à «ces voix innombrables qui l'habitent» $(R L, \mathrm{p} .4 \mathrm{I})$, l'écrivain choisit de se mettre en scène dans un espace d'écriture conçu comme manipulation du réel, du lecteur et du narrateur-écrivain. L'imaginaire que découvre alors la fiction est invention d'un "dire», où le chassé-croisé des voix narratives démonte les rouages du processus d'écriture au sein duquel les personnages non anthropomorphes errent dans une longue coulée verbale qui donne droit de cité au rêve, au délire. Dans cette communication (évoluant parfois en foire d'empoigne) avec "Ain», alias "Hdiddane», alias "E. T.», s'édifie alors l'image d'un nouvel être-en-écriture qui se fraye un chemin d'existence

I2. A. Laâbi, Les Rides du lion, Messidor, 1989 (désormais $R L$ ). 
dans les méandres d'un "délir[e] en plusieurs langues avec des poèmes sauvages et incongrus" ( $R L$, p. I5).

Écriture jouissive, en jaillissement, orchestrée par "la bataille des voix" ( $R L$, p. I27) autorisant cette aventure textuelle qui « ne parodie plus le monde mais l'invente $»^{{ }^{3}}$. Et dans ce télescopage des voix, des sentences qu'elles profèrent, s'abolissent toutes les prisons, tous les pouvoirs qui tendent à limiter la liberté d'expression et la responsabilité de l'écrivain :

[...] La mythomanie semble être le propre de l'écriture et je me demande, après l'expérience que nous avons vécue, si le rire est vraiment le propre de l'homme. Je crois même que tu as fini par comprendre que mon apport a été salutaire. J'en avais assez de te voir prosterné devant le cul du lecteur. Alors j'ai fait respirer ton texte comme j'ai pu [...] (RL, p. 172-173)

Ce faisant, l'écriture fictionnelle se veut instrument d'ivresse et préfere l'humour au pathos pour tourner en dérision l'absurdité de «ce pays [qui] peut en cacher un autre» et l'inanité d'une foi infaillible en ses propres exigences esthétiques.

\section{Dé-parler la langue. Une esthétique du verbe libre, du mot-projectile}

Lire les auteurs maghrébins aujourd'hui amène aussi, de plus en plus, à grimper dans un carrousel verbal vertigineux charriant de multiples et riches réminiscences, les mille bruits de l'existence dans son actualité tragi-comique, où le pouvoir des mots qui fustigent, qui recréent, tisse un lien de complicité avec le lecteur. Changer les mots pour changer sa relation au monde, telle semble être la gageure soutenue par Abderrahmane Lounes, Mohamed Saïd Fellag, Aziz Chouaki, pour ne citer que quelques-uns des prestidigitateurs du langage chez qui les mots font l'humour dans une entreprise d'encanaillementmanipulation de la langue qui vaut le détour. Dans une perspective moins spectaculaire que celle de Fellag ou Abderrahmane Lounes, Aziz Chouaki, romancier et dramaturge, exploite le verbe mordant d'une jeunesse citadine grâce au mélange des tonalités comique et tragique.

Interrogé sur son activité littéraire et sur son choix d'écriture, il répond :

J'écris en français, certes, histoire oblige, mais à bien tendre l'oreille, ce sont d'autres langues qui parlent en moi, elles s'échangent des saveurs, se passent des programmes télé, se fendent la poire. Il y a au moins, et surtout, le kabyle, l'arabe des rues et le français ${ }^{14}$.

I3. L. Aragon : "Jusqu’ici les romanciers se sont contentés de parodier le monde. Il s'agit maintenant de l'inventer.»

I4. El Watan, Io mai 2007, p. 24. 
Dans L'Étoile d'Algeris, poésie, musicalité et humour s'allient à ces langues pour recréer la parole vraie du quotidien, où l'humour et l'autodérision imposent leur loi pour faire du langage l'écho tout à la fois des plaies du temps et des espérances aspirant à les juguler. Pour n'évoquer ici que quelques exemples, en liaison avec le procès fait à l'islamisme, retenons :

[...] c'est comme ça que tu tombes islamiste, c'est quand tu fatigues.

De rêver, d'aimer, de vivre. Les meilleurs copains sont tombés comme ça, à force de fatiguer, tu peux plus rien voir venir. (EA, p. 19)

[...] Fatigué, Moussa laisse tomber, oui c'est comme ça que ça commence sûrement, quand tu fatigues. (EA, p. 9I)

Véritables mots-programme de la fiction, les verbes «tomber» et "fatiguer» (traduction littérale de l'arabe dialectal pour signifier l'impuissance à remédier au fatum) résonnent du mal-être de cette "Algérie [qui] devient folle» comme dirait Boualem Sansal, de cette jeunesse récupérée par l'idéologie pernicieuse tristement à la mode. Ils propulsent aussi une parole spontanée, lieu de l'impulsion et d'un cryptage fantaisiste du sens qui se joue de l'image funeste de cette nouvelle Algérie défigurée, où il ne fait plus bon vivre quand on est homme de culture, qu'on ne porte pas «barbes, kamis, comme toute l'Algérie, format national standard» (EA, p. 67).

Divertissant est ce parler, mais pas irresponsable, puisqu'il renvoie au lecteur l'image d'une génération en mal d'idéal, à laquelle on ne peut s'identifier sans se rebeller contre soi-même, contre le sort et contre les instigateurs du mal nommément identifiés : "Abassi Madani et Ali Benhadj, fin du monde, État islamique.» ( $E A$, p. 66) L'écriture qui transcrit sur le vif le langage de cette génération résonne d'un absurde à rire et à pleurer, que traduit d'ailleurs le parcours même du personnage central du roman, dans sa dynamique onomastique. De Méziane Boudjiri, son nom réel, à Moussa, diminutif de Massinissa, son héros berbère, puis à Massy, son nom de star montante, et enfin à Nour, son nom de guerre d'Émir, membre éminent du GIA, il y a programmation de la tragi-comédie de son existence d'ailleurs pressentie par la prédestination de sa naissance : «Moussa est né un premier avril, un canular de la nature» ( $E A$, p. 94). Au travers de l'ascension rêvée, c'est l'histoire d'une chute irréversible qui nous est racontée.

Dans le recours à l'humour, chez Chouaki comme chez les auteurs cités plus haut, l'arrimage de l'écriture à la parole de la rue ne procède pas d'un encanaillement stérile de la langue, mais entend mener à une mobilisation véritable qui déjouerait tous les tabous, transcenderait le tragique. Et cette récupération des énoncés du vécu recyclés dans un processus créatif affranchi

I5. A. Chouaki, L'Étoile d'Alger, Balland, 2002 (désormais $E A$ ). 
des règles de l'énonciation courante, en engendrant un effet jouissif, car transgressif et pluri-vocal, se veut solidaire d'un lectorat "élitiste» spécifique : celui, populaire et nombreux, des initiés de «l'oraliture». Cette littérature-là, libérée du diktat du "bien écrire» enseigné, redéfinit ses assises et ses ambitions et, ce faisant, élève à sa façon le langage. Il ressort de cette lecture panoramique deux évidences : la première est que le désespoir est mobilisateur; la seconde est que chez tous les écrivains maghrébins, aujourd'hui, les plaies du vécu - leitmotive obsédants référant à une errance multiforme, au questionnement de soi et du monde - sont prétextes à un travail créatif donnant accès à un champ d'une grande fertilité. La recherche esthétique, en effet, prend le pas sur la description du réel. Elle apprivoise la violence du présent, construit, grâce aux artifices du langage, un processus de désenchantement subtilement porteur d'un enchantement nouveau, permettant de croire au changement et de réinventer - pour ainsi dire - le monde :

Si par l'art, nous pouvons expérimenter quelque chose, nous pourrions peut-être devenir capables de changer notre avenir ${ }^{16}$.

Gageons que le livre maghrébin de demain n'a pas fini de mettre en place de nouveaux dispositifs d'écriture, "[...] livre de magie qui opère comme un manuel de guérilla ${ }^{17}{ }^{\prime}$.

I6. S. Kane, Lexi-textes 3, I998, p. I98.

I7. F. Badre, L'Avenir de la littérature, Gallimard, 2003, p. I88. 